\title{
SLOVENE SOCIETY
}

\section{SS-01}

\section{Value of transvaginal hydrolaparoscopy in infertility} evaluation

M. Reljič, V. Vlaisavljević, V. Gavrić-Lovrec

Gynaecologic Clinic, University Clinical Centre Maribor, Maribor, Slovenia

Objective: In 1998, transvaginal hydro laparoscopy (THL) was introduced and described as a first line technique for the exploration pelvic cavity in infertility women. The aim of our retrospective study was to evaluate the usefulness and prognostic value of THL.

Design §Methods: Consecutive patients undergoing THL for infertility between 2001 and 2006 were included in the study. The main outcome measures were the rate of the successful access to the pouch of Douglas, the rate of complications, findings in term of tubal pathology, pelvic adhesions, and endometriosis. Laparoscopy was followed immediately after THL in all cases with pathological findings and concordance of both techniques were evaluated. Data on fertility outcome were collected by reviewing medical records only in patients with normal THL findings. Results: A total of 167 women without obvious pelvic pathology were included. The rate of successful access to the pouch of Douglas was $98.8 \%$. Apart from three cases of transitional febrile condition we had no complications. The rate of complete evaluation of all the pelvic structures with THL was 86.7 (143/165). The incomplete visualization was found to be related to the extensive adhesion in the pelvis. In 112 patients $(67.9 \%)$ the THL procedure has shown a morphological normal pelvic examination with at least one patent tube. There were some abnormalities seen in the remaining 53 patient as follows: 45 patients had adhesions, 22 endometriosis and 7 double sided tubal occlusion. The morphologically findings using THL and laparoscopy were strictly concordant in 42 patients $(79.2 \%)$. The concordance in tubal patency was $88.6 \%$. Discordances were always limited to one tube. In all cases the discordant findings were considered not to have any clinical impact.

Among 112 patients with normal THL findings 47 (42.0\%) conceived spontaneous or after intrauterine insemination. Conclusion. THL is a minimally invasive, safe and accurate procedure that may be considered as an alternative to diagnostic laparoscopy in the routine assessment of infertile patients without obvious pathology.

\section{SS-02}

\section{Office hysteroscopy in Slovenia}

B. Žegura, I. But, I. Takač

Gynaecologic Clinic, University Clinical Centre Maribor, Maribor, Slovenia

Objective. Office hysteroscopy (HSC) has become an indispensable procedure in diagnosis and treatment of pathology of uterine cavity and cervical canal. In comparison to D\&C of the uterus, HSC makes it possible to examine the uterine cavity and cervical canal with targeted biopsies or removal of focal lesions. Focal lesions, such as endometrial and cervical polyps, myomas, focal endometrial hyperplasia and initial endometrial carcinoma, might be missed by $\mathrm{D} \& \mathrm{C}$, which is considered history as the chosen method in detecting pathology of the uterine cavity and cervical canal.

Methods and Results. Since July 2003, when office HSC was introduced in Slovenia, till May 2007, 912 women (52.4 \pm 12.3 years) visited our outpatient HSC office because of bleeding disorders $(56.1 \%)$, abnormal uterine cavity $(89.6 \%)$, infertility (7\%), missed IUD (5.3\%) and persistent gestational tissue $(3.8 \%)$. In $87.5 \%$ the procedure was performed by the vaginoscopic approach; in the remaining patients we could not avoid dilatation of cervical canal under local anesthesia. Office HSC was successful in $96.3 \%$ of cases. Reasons for failure were occlusion of the cervical canal (3.8\%), poor hysteroscopy view $(1.1 \%)$ and severe discomfort (1\%). Failed cases were referred for investigation under general anesthesia. $0.87 \%$ experienced vasovagal attacks immediately after the procedure. There were no other complications related to the use of local anesthesia or the HSC itself. The uterine cavity and cervical canal appeared normal in $39.4 \%$. In $47.3 \%$ we found endometrial polyps, in $10 \%$ myomas and in $1.8 \%$ severe intrauterine adhesions. Intracervical pathology was represented with cervical polyps $(4.1 \%)$ and adhesions $(1.9 \%)$. Targeted endometrial biopsies were taken in $61 \%$. The histology showed normal endometrium (33.6\%), endome- 
trial polyp (54.3\%), endometrial hyperplasia (8\%) and atypical endometrial hyperplasia $(0.3 \%)$. In $2.8 \%$ we detected endometrial carcinoma, in one patient clear cell endometrial carcinoma and in one uterine sarcoma. 53.8\% of women with uterine malignancy were asymptomatic. Endometrial biopsy failed to produce sufficient tissue $(1.6 \%)$ for complete histological diagnosis more often in women with an atrophic cavity at HSC $(p<0.0001)$. Operative procedures were performed in $43.3 \%$ : polyp ablation (27.3\%), septum resection (8.7\%), removal of IUD $(5.3 \%)$, myomectomy $(2.1 \%)$, synaechiolysis $(2.1 \%)$ and 5 Essure sterilizations. In $69.2 \%$ office HSC was a "see and treat procedure". The pain experienced during HSC was recorded with a visual analogue scale (VAS). The vaginoscopic approach compared to cervical dilatation under local anesthesia was associated with less pain $(1.2 \pm 1.4$ vs. $2.1 \pm$ $1.5, \mathrm{p}<0.000001)$.

Conclusions. Office HSC is an accurate and reliable method for diagnosis of intrauterine and cervical disorders. Its combined diagnostic-therapeutic approach makes it a simple, fast, safe and patient friendly procedure with low costs.

\section{SS-03}

The value of hysteroscopy in the diagnosis of the cause of uterine bleeding in perimenopausal women

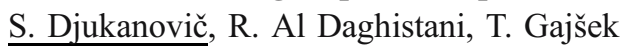

General Hospital Ptuj Dr. Jože Potrč, Gynaecological Department, Ptuj, Slovenia

Objective: Hysteroscopy is an important method in the diagnosis of the causes of abnormal uterine bleeding in perimenopausal patients. In most gynecological institutions a routine methods dilation and curretage (D\&C) is still » the golden method « for diagnosis of uterine bleeding in patients at the menopause. In our retrospective study we made a correlation between hysteroscopy and D\&C in the diagnosis of the causes of the uterine bleeding at the mentioned population. The aim of the study is to find if hysteroscopy can replace of the $\mathrm{D} \& \mathrm{C}$ as a routin diagnostic method.

Design \& Methods: In the retrospective study we included 257 patients who were hospitalised due to uterine bleeding from the begining of the year 2000 to the end of the year 2004. Patients have been devided into two groups. In group A we included patients who had hysteroscopy. In group B (control group) were patients who underwent D\&C. Results that were collected from the patients files and from their histopathological findings. The results were statistically evaluated. In both groups disclusion criteria have been taking into account.

Results: There was no statistical difference according to the age, body mass index, duration of the symptoms and number of deliveries. We diagnosed significantly higher number of endometrial polyps in group A than in group B. There was significantly higher recurrence of uterine bleeding in group B.The duration of the procedure, pain during the procedure and the late complications between the two groups were not statisticaly significant.For the diagnosis of endometrial carcinoma hysteroscopy apeared to be the more reliable method.

Conclusions. Diagnostic hysteroscopy is equally effective or even superior method for the diagnosis of the causes of uterine bleeding to D\&C. With hysteroscopy less number of additional procedures due to recurrent uterine bleeding is required. In case of acute and severe uterine bleeding hysteroscopy is not possible. D\&C is still the golden standard for the diagnosis of uterine bleeding but it has to be combined if possible with hysteroscopy.

\section{SS-04}

Hysteroscopy in the department of gynaecology and obstetrics Novo Mesto in the period from 2004 to 2006 our experiences

A. Pišek, M. Gradecki

General Hospital, Department of Obstetrics and Gynaecology, Novo Mesto, Slovenia

Background: Hysteroscopy has due to its many advantages in everyday practice replaced a large portion of dilatation and curettage as diagnostic and therapeutic procedure of the pathology of the uterine cavity. In the period from 1996 to 2000 the portion of hysteroscopies among surgical procedures inside uterine cavity was $20 \%$ while in the period from 2001 to 2006 its proportion has risen to $46 \%$. Our objective was to present the number and structure of those procedures that represent the everyday practice of our department.

Design\&Methods: We reviewed the hysteroscopies that took place in our department during the period from 2004 to 2006. The data were obtained and processed with help of the Birpis 21 medical informational system.

Results: In the period from 2004-2006 619 hysteroscopies were performed. In the same period 721 dilatation and curettages were done (we excluded procedures done in connection with pregnancy and abortion). $21 \%$ of hysteroscopies were listed as diagnostic procedures while $79 \%$ were operative. Among the operative hysteroscopies 52\% represent resections of endometrial polyps, 17\% endometrial ablations, $16 \%$ hysteroscopic myomectomies and $12 \%$ resections of uterine septa. The rest were done for various less frequent reasons such as for instance dissection of intrauterine synaechiae or late removal of residual placental tissue after birth or abortion. In the observed period we had 
no major complications such as uterine perforation or bleeding that would require operative revision and no complications connected with the fluid overload. We had only five readmissions for treatment of suspected or proven infection after hysteroscopy.

Conclusion: In our institution hysteroscopy has won its place as the method of choice for diagnosis and treatment of the pathology of the uterine cavity and has been proven as safe and efficient method of treatment.

\section{SS-05}

\section{Hysteroscopic resection of small uterine septum improves implantation in IVF}

T. Tomazevic, H. Ban Frangez, I. Virant-Klun, M. Ribic Pucelj, A. Vogler, B. Zorn, S. Drobnic, E. Bokal Vrtacnik, L. Kermavner Bacer, B. Valentincic, J. Mivsek

University Clinical Centre, Department of Obstetrics and Gynaecology, Ljubljana Slovenia

Objective Recently the data were generated showing that hysteroscopic resection of small uterine septum (AFS 6) improves implantation in IVF. In order to further test these findings we retrospectively analysed the IVF/ICSI results in a larger non historical case control study.

Design and Methods Embryo transfers (ETs) in 768 IVF/ ICSI stimulated cycles performed before and after hysteroscopic metroplasty in larger (AFS grade 5) and in smaller (AFS grade 6) uterine septa were analysed and compared to 1624 matched controls. Two subsequent ETs in IVF/ICSI stimulated cycles performed in women without uterine malformations were included as controls. Logistic regression and chi square test were used for statistical evaluation. Results The pregnancy/ET rates before surgery in women with AFS 5 septa and AFS 6 septa were similar (12\% and $14 \%$ respectively). and significantly lower compared to pregnancy/ET rates in the controls without uterine malformations $(29 \%$ and $26 \%$ respectively) $(\mathrm{P}<0,001)$. After surgery in women with AFS 5 septa and AFS 6 septa the pregnancy/ET rates were similar ( $23 \%$ and $26 \%$ respectively) and did not differ to the results in the two control groups without uterine malformations $(26 \%$ and $28 \%$ respectively) ( $\mathrm{P}<0,3$ and 0,8 respectively).

Conclusions. By decreasing the implantation rate the AFS 6 as well as AFS 5 uterine septa negatively influence the success in IVF and ICSI $(\mathrm{P}<0,001)$. The small uterine septum should therefore not be considered a variant of normal anatomy but should be treated before IVF is attempted.

\section{SS-06}

Endometrial ablation with thermal balloon

M. Ribič-Pucelj, B. Cjetićanin, B. Grulovič

University Clinical Centre. Department of Obstetrics and Gynaecology Ljubljana, Slovenia

Objective: The present indications and efficacy of thermal balloon (TC) (Thermachoice, Gynecare) endometrial ablation in terms of reduced menstrual bleeding and dysmenorrhoea and to compare the results with transcervical resection of endometrium (TCRE).

Design \& Methods: Between 1st September 2000 and 31st December 2004, 308 patients were admitted to the Department for endometrial ablation due to dysfunctional uterine bleeding. Two hundred forty one $(78 \%)$ responded the questionnaire: $112(85 \%)$ who underwent TC and 129 $(79 \%)$ in whom TCRE was performed. The indication for TC was failed, intolerable or contraindicated hormonal treatment, uterine size $\leq 10 \mathrm{~cm}$, uterine pathology excluded with vaginal ultrasound examination, evaluation of the endometrium by D\&C, hysteroscopy or hysteroscopy and endometrial biopsy. No preoperative preparation of endometrium was used. All the patients received preoperatively 1 ampoule of nonsteroid anti-inflammatory drug i.m. TC was performed according to the prescribed procedure. Fifty five percent of the procedures were performed in local anaesthesia while $45 \%$ required i.v. analgesia. All the patients at high risk for surgery and/or anaesthesia, but with complete preoperative work-up, were included in TC group. TCRE was performed in general anaesthesia after preoperative preparation of the endometrium. in these patients hysteroscopy was diagnostic and therapeutic procedure at the same time.

Results: The efficacy of both procedures was evaluated in 2006 when there was at least 1 year after the procedure. Following TC amenorrhea was achieved in $26,4 \%$ patients and was significantly higher than in patients who underwent TCRE in whom it was achieved only in $4,4 \%(\mathrm{p}<$ 0.05 ). Hypomenorrhea following TC was achieved in 32, $7 \%$ patients, eumenorrhea in $31.8 \%$ while failure rate was $9,1 \%$ and was comparable to TCRE where it was $36.0 \%$ and $59.0 \%$ and $8,8 \%$ respectively. Dysmenorrhea was significantly decreased in both groups. There were no major complications.

Conclusions: TC has, beside higher amenorrhea rate several other advantages: short learning curve, no need for experiences in hysteroscopic surgery, no risk for surgical complications, no need for preoperative preparation of the endometrium, most of the procedures can be performed under local anaesthesia on an outpatient basis. The disadvantage is high costs of the balloon, which is in our 
country not yet reimbursed by social insurance and postoperative pain occurring in about $17 \%$ of patients.

\section{SS-07}

Efficacy of hysteroscopic resection of endometrium in the treatment of dysfunctional uterine bleeding N. Brus, M. Ribič-Pucelj

General Hospital Celje, Department of Obstetrics and Gynecology, Celje, SloveniaUniversity Clinical Centre, Department of Obstetrics and Gynecology, Ljubljana, Slovenia

Background. Irregular uterine bleeding is the most common problem in outpatient clinic. Fifty to $60 \%$ of all irregular uterine bleeding are dysfunctional. Hysteroscopic resection of endometrium (TCRE) is efficient therapy in 80 to $90 \%$ of patients with dysfunctional bleeding. We designed the study to determine effecacy of hysteroscopic resection of endometrium between 2 to 6 years after hysteroscopic operation.

Design\&Methods. One hundred twenty nine women following hysteroscopic resection of the endometrium between January 1998 and December 2002 at the Department of Obstetrics and Gynecology in Ljubljana were included in the retrospective study. We evaluated the efficacy of hysteroscopic resection of endometrium: improvement of menorrhagia and dysmenorrhea, patient's satisfaction with the procedure, number of eumenorrhoic, hypomenorrhoic and amenorrhoic patients after the procedure. The procedure was performed immediately after menstruation or after medical preparation of the endometrium. Hysteroscopic resection of the endometrium was performed with $9 \mathrm{~mm}$ resectoscop, 5\% glucose as distension medium, and in general anesthesia.

Results. Following TCRE menorrhagia was improved in 120 $(94,5 \%)$ and dysmenorrhea in $81(86 \%)$. One hundred eleven $(87,4 \%)$ patients were satisfied with the procedure. Operation failed in 7 patients $(5,5 \%)$. In 5 patients hysterectomy was made and in 2 patients repeat TCRE was successful. In the six years period the efficacy of the treatment was increasing until 5 -th year after the procedure, then recidives appeared Preoperative medical preparation of the endometrium was made in $41(32,3 \%)$ patients In $5(3,9 \%)$ patients hysterectomy was performed. Repeat operation was successful in 2 out of 3 patients Complications after the procedure were: endometritis, early postoperative bleeding from the uterus and minor fluid overload in 2 patients.Avarage age of the patients at the time of treatment was 44, 6 years, 19 of them in 6 years after the treatment became menopausal. Conclusions. Hysteroscopic resection of the endometrium is very successful method for the treatment of dysfunctional uterine bleeding. Advantage of the procedure compared to the other techniques of endometrial ablation is possibility of histological examination, short hospitalization and quick recovery. Preoperative preparation of the endometrium and experienced surgeons can improve the results.

\section{SS-08}

Incidence of malignant endometrial polyps in women in reproductive, perimenopausal and menopausal periods E.Vrtačnik Bokal, M. Ribič-Pucelj, B. Cvjetićanin

University Clinical Centre Ljubljana, Department of Obstetrics and Gynaecology, Ljubljana, Slovenia

Objective: Endometrial polyps are frequent in all groups of women with the incidence ranging from 16 to $34 \%$. The highest incidence of malignant polyps is found in postmenopausal women experiencing uterine bleeding. The aim of this retrospective study was to analyse the incidence of malignant polyps in women in their reproductive, perimenopausal or postmenopausal period.

Design \& Methods: Analysis involved 320 histological diagnoses of endometrial polyps of women who were treated at the Department of Obstetrics and Gynecology in Ljubljana in the time period 2005-2006.

Results: Of the 117 polyps of women in reproductive period, $114(97.4 \%)$ were benign, 2 (1.7\%) hyperplastic, and $1(0.9 \%)$ malignant; of the 79 polyps of women in perimenopausal period, $78(98.7 \%)$ were benign, and $1(1.3 \%)$ malignant, and of 124 postmenopausal polyps, $116(93.6 \%)$ were benign, 7 (5.6\%) hyperplastic, and $1(0.8 \%)$ malignant.

Conclusion: Endometrial polyps are relatively commonly detected in women of all age groups, which is due to improved diagnosis using transvaginal ultrasound. Although the reported incidence of malignancy in recent reports as well as in ours is low, operative hysteroscopy should be offered to symptomatic women and to asymptomatic women with the following risk factors: advanced postmenopause, hypertension and polyps $>1.5 \mathrm{~cm}$. Due to improved technology, the procedure can be done in an office setting, with little risk and without significant patient discomfort.

\section{SS-09}

Should asymptomatic endometrial polyps be resected? B. Verdnik Golob

General Hospital Slovenj Gradec, Department of Gynecology and Obstetrics, Slovenj Gradec, Slovenia

Background. The prevalence of endometrial polyps (EP) is high $(25 \%)$, but the risk of malignancy low $(0.5-4,8 \%)$. Women are often asymptomatic. More EP are diagnosed 
with the use of transvaginal ultrasound scanning. The purpose of this study was to determine whether clinical risk factors and symptoms can predict their histopathological characteristics and whether is it safe to leave the asymptomatic EP in uterus.

Design\&Methods. Retrospective study of 365 women with removed EP in the years 2001-2007 was done. 52, 9\% patients underwent operative hysteroscopy, $47,1 \%$ uterine curettage. Clinical data were obtained from patients' medical reports. EP were subdivided into groups as symptomatic A and asymptomatic B; benign (benign, hyperplastic without atypia) and malignant (hyperplastic with atypia, cancerous). Statistical analysis was performed. Differences between groups were evaluated using MannWhitney test. As a measure of bivariate correlation the Spearman Rho was used, with $\mathrm{p}<0.01$ considered to be statistically significant.

Results. In 285 patients (70, 7\%) EP were symptomatic, in $107(29,3 \%)$ asymptomatic. In group A 2 hyperplastic EP with atypia and one cancerous EP $(0,8 \%)$ were found. In group $B$ all were benign. Patient age $(p<0.01$, positive correlation) and menopausal status $(\mathrm{p}<0.01$, negative correlation) were associated with symptoms significantly. Hypertension showed significant association with malignant EP $(\mathrm{p}<0.01)$.

Conclusions. The incidence of malignancy is low. Symptoms were correlated with age, in postmenopausal women more likely asymptomatic. Hypertension is associated significantly with malignant degeneration of EP. Asymptomatic EP were all benign but not significantly. Histopathology remains the golden standard. Their removal (hysteroscopic) would be rationale, especially in hypertensive women.

\section{SS-10}

Laparoscopic supracervical hysterectomy - a treatment option for women with symptomatic uterine fibroids and adenomyosis

I.But, B. Žegura, M.Reljić, S.Rakić, M. Pakiž, M.Rebernik Milić, S. Ciglar S

Gynaecologic Clinic, University Clinical Centre Maribor, Maribor, Slovenia

Objective: Laparoscopic supracervical hysterectomy (LSCH) is a minimally invasive endoscopic procedure which represents a real alternative to abdominal hysterectomy (AH) and is due to lesser invasiveness and fewer complication rate also recommended by Cochrane Review. We are presenting our experiences with LSCH procedures which have been performed at our department since 2005 .
Design and Methods: LSCH was performed in 40 symptomatic patients who were scheduled to have AH. We followed the surgical time and possible perioperative complications. Patients were divided in two groups according to the diagnosis (uterine fibroids, adenomyosis) and according to the weight of the uterus (threshold $210 \mathrm{~g}$ ). For statistical analysis descriptive and nonparametric statistics were used. Statistical significance was set at $\mathrm{p}<0.05$.

Results: The average age of patients was 47 years. The average surgical time was $84.5 \mathrm{~min}$ and the average intraoperative blood loss was $266.4 \mathrm{ml}$ (20.0 to $1000.0 \mathrm{ml})$. The average weight of the uterus was $292.7 \mathrm{~g}$ (median $210 \mathrm{~g}$ ). There were no conversions to laparotomy. We had only few technical problems with instruments which made our surgical time longer. The average duration of the hospitalization was 2.6 days ( 2 to 6 days).

Eighteen patients (45\%) had uterine fibroids and 22 $(55 \%)$ had adenomyosis. Patients with uterine fibroids had significantly heavier uterus $(\mathrm{p}=0.01)$ and the surgical time was longer ( 95 vs. $70 \mathrm{~min}, \mathrm{p}=0.007$ ). The surgical time correlated to the weight of the uterus $(\mathrm{r}=0.81 ; \mathrm{p}=$ 0.000 ), especially when uteruses were heavier than $210 \mathrm{~g}$ (95 vs. $60 \mathrm{~min}, \mathrm{p}=0.000$ ). The blood loss correlated to the weight of the uterus $(\mathrm{r}=0.675, \mathrm{p}=0.000)$, and was higher with heavier uteruses $(270.0$ vs. $80.0 \mathrm{ml}, \mathrm{p}=$ 0.000).

Conclusions: Our results show that LSCH is a safe procedure and is according to our experience comparable to $\mathrm{AH}$ in terms of surgical time and blood loss but has shorter hospitalization and rehabilitation time. However, blood loss and surgical time are connected to weight of the uterus and we should present this problem to patients before the surgery. Our surgical time could be improved with having better instruments (electric-power-driven instead of hand-driven morcellator), which would not broke during the operation so often (harmonic scalpel failure, blunt morcellator knive).

\section{SS-11}

Laparoscopic myomectomies in General Hospital Celje in the years 2001 to 2006

K.Jakopič, V. Weber

General Hospital Celje, Department of Obstetrics and Gynaecology, Celje, Slovenia

Objective: Myomas are benign tumours arising from smooth muscle cells of the uterus that occur in about $25 \%$ of all women in reproductive age. Mostly they are asymptomatic, but some can cause bleeding disorders, pain, 
pelvis pressure and infertility. There is an increasing demand for conservative treatment due to a rise in the average age of primiparas and the consequent need to preserve reproductive function. Modern tendency towards less invasive treatment has made laparoscopic myomectomy one of the most prominent surgical approaches. In our retrospective study we have analysed perioperative results of all laparoscopic myomectomies performed in our hospital in the years 2001 to 2006 .

Methods: In the study we included all patients in whom we started operation with laparoscopic approach and were diagnosed with myomas prior to procedure in between 2001 and 2006. All operations were performed under general anaesthesia. We used Zeiss optics and injected diluted vasopressin into myometrium prior to incision with monopolar needle. We extracted myoma using two graspers, sutured uterus with Vicryl and removed myoma from abdomen using morcellator. In the study we compared preoperative care, procedure duration, size, number and location of myomas, complications during operation and hospital stay, histology reports and duration of hospital stay.

Results: In the years 2001 to 2006 we preformed 239 laparoscopic myomectomies. All patients received pre- and perioperative prophylactic anticoagulation therapy with LMWH. Average age at the time of the procedure was 40.07 years. We operated on subserous and intramural myomas up to $9 \mathrm{~cm}$ in diameter. In 12 cases conversion to laparatomy was needed due to uncontrolled bleeding or size and position of myomas. There was no case of bowel or large vessel injury. All histological results were negative for malignancy. Average dismissal time was day 3 after procedure.

Conclusions: LPSC myomectomy is an excellent method for a less invasive surgical removal of myoma. With more experience and better equipment we operate on bigger and bigger myomas, even those with intraligamentary and isthmic locations. In the future the number of LPSC myomectomies will increase even more as indications for LPSC approach will no longer be limited with our surgical skills, experience or equipment.

\section{SS-12}

\section{Our experience in laparascopy in prepubertal girls A.Štolfa, B. Požlep \\ University Medical Centre, Department of Obstetrics and Gynecology, Ljubljana, Slovenia}

Objectives: Laparoscopic diagnostic and operative treatment is gaining acceptance in pediatric population. Indications for application of laparoscopy in this age group are: suspected adnexal torsion, ovarian cysts, pelvic pain and rare genital anomalies.

Design: We have performed 10 to 15 laparoscopies per year in teenagers up to 18 years of age in the last years. We have a lot of experience in adolescents, since the procedure is technically similar to that in grown-up women. We started to perform laparoscopies in girls before puberty only some years ago. Besides the fact that there is not much gynecological pathology in young girls, mostly pediatric surgeons meet it.

Results: The first procedure in prepubertal girl has been done in a 8-year-old girl for a diagnostic purpose perityphlitic abscess was found. Eight other laparoscopic procedures in 5 to 10 -year-old girls have been done in the last three years: 5 cases of a successfull detorsion of the adnexa, an enucleation of a benign ovarian cyst, a removal of the adnexa and staging after the enucleation of a teratoma that had some immature components, and a removal of completely necrotic cystic adnexa.

Conclusion: It is evident also from the literature that the majority of young patients of the gynecologic authors are adolescents and they only quote solitary cases in girls before the age of 10. A laparoscopic technique using an adjusted equipement is a possible choice of treatement in prepubertal girls, however it is important that the procedure is attempted only by a well trained team.

\section{SS-13}

Conservative vs. radical surgical treatment of ectopic pregnancy in women desiring further fertility

M. Ribic-Pucelj, S. Dobrecovic

Department of Obstetric and Gynecology, University Medical Centre Ljubljana, Ljubljana, Slovenia

Objectives. To evaluate fertility following laparoscopic surgical treatment of ectopic pregnancy (EP) in women desiring further fertility and to compare pregnancy rates after salpingostomy and salpingectomy.

Design and Methods. Retrospective study performed in a tertiary care university centre. In the time period from 1994 to 2000,801 women underwent laparoscopic surgical treatment of EP; 531 fulfilled the criteria for the study the possibility of spontaneous conception, less than 38 years of age and desire for further fertility. They were sent a selfadministered questionnaire; $361(74.2 \%)$ responded, and 268 women who attempted to conceive, were enrolled in the study. Salpingostomy was performed with monopolar needle without suturing the tube and salpingectomy with bipolar forceps and scissors. Most of the procedures were performed by residents and younger gynecologists not experienced in tubal surgery, therefore the scoring system 
for surgical treatment was, unfortunately, not always taken into account. The data were statistically analysed using Chisquare test, Student t-test and odds ratio.

Results. Spontaneous pregnancy occurred in 142 (55.9\%) women, in 55 (75.3\%) following salpingostomy and in 87 $(69.0 \%)$ following salpingectomy. The pregnancy rate was higher after salpingostomy, but not statistically significantly. In the salpingostomy group the delivery rate was $58.2 \%$, the spontaneous abortion rate $14.5 \%$, the repeat EP rate in the same tube $14.5 \%$ and in the contralateral tube $12.7 \%$, and in the salpingectomy group $59 \%, 9.2 \%$, respectively, while EP in the remaining tube occurred in $26.4 \%$ of women.

Conclusions. Laparoscopic surgical treatment of EP is effective resulting in over $50 \%$ intrauterine pregnancy rate. Despite widespread assisted reproductive technologies, there is still place for conservative surgical treatment of EP, particularly in younger women and in women with a single tube, but to achieve even better results, experience in tubal surgery is recommended.

\section{SS-14}

Incontinence surgery - towards minimal invasiveness A. Lukanovič, M. Barbič

Department of Obstetrics \& Gynaecology, University Clinical Centre Ljubljana

Objective: When speaking of surgical treatment of female stress urinary incontinence we have to admit that there was an enormous development done in the last decades. The latest, the third generation named TVT Secure is another step towards minimal invasiveness as it requires only a small vaginal incision and no exit points.

Design \& Methods: Due to advantages and cost effectiveness, TVT operation has mostly substituted other surgical approaches for the treatment of female SUI at our Department. Prior the surgery each patient underwent the diagnostic protocol including aerodynamic measurement. Since January 1998 we have operated at our Department 1656 patients. First 60 operations we have done in general or spinal anaesthesia, the rest in local. In the time period 1998 to march 2005 there were 1383 TVT retro pubic operations. From March 2005 to December 2006 there were 267 TVT O operations and from January to May 200744 TVT S operations. Even we did not have any major complications with TVT of first and second generation we switched to TVT S in January this year as we believe that due to micro invasiveness and benefits the patients accept it better.

Results: Postoperative continence rates 6 weeks after surgery are promising. We have an overall success rate of
$95 \%$ for primary and $89 \%$ for recurrent operation. The time spent per TVT $\mathrm{S}$ procedure was less than 10 minutes. We had no severe complication as rejection of tape, vascular or neuromuscular damage. The percentage of tape erosions is 2, 4\%. All were successfully closed by suturing excised vaginal mucosa. With learning curve the problem with tight tape and urinary retention is no longer disturbing. As the surgical approach is less and less invasive, there is quicker recovery time and evident shortening of hospital stay and all patients after TVT S were released from Department first post operative day.

Conclusions: Undoubtedly this surgical technology is developing in the right direction. We are looking for procedures which have high continence rates and are bringing better responses from patient' side. Last but not least this is a cost saving procedure to treat female SUI. Such approach offers us the possibility to treat a wider population of incontinent patients with this proved more successful and well tolerated procedure.

\section{SS-15}

Anterior Transobturator Mesh (ATOM) and/or Posterior Ischiorectal Mesh (PIRM) for correction of female pelvic organ prolapse and pelvic floor dysfunction

M. Lužnik

General Hospital Slovenj Gradec, Department of Gynecology and Obstetrics, Slovenj Gradec; Slovenia

Objectives: Benefit of alloplastic mesh implantations with needle applicators for correction of female pelvic organ prolapse and pelvic floor dysfunction is in their minimally invasive intervention to achieve a marked improvement in pelvic organ static and pelvic floor function. For younger women it is often very important to preserve the otherwise healthy uterus and normal volume of vagina. All these demands can be fulfilled with an anterior transobturator mesh - ATOM and/or a posterior ischiorectal mesh - PIRM procedures.

Design \& Methods: In the first year of practicing this procedure, between April 2006 and April 2007, we performed sixty-two operative corrections of female pelvic organ prolapse and pelvic floor dysfunction with mesh implants on our Department of Gynecology and Obstetrics. The twenty-five patients with surgical procedure TVT-O or Monarc as solo intervention are not added to this number.

On 8 out of 62 patients hysterectomy was performed concomitantly, only for gynecological indications other than prolapse itself.

On 38 out of 62 patients prolapse was corrected with mesh and uterus was preserved. 
In 16 cases out of 62 , correction of vaginal stump prolapse after hysterectomy was performed in 3 cases with ATOM and PIRM, in 8 only with ATOM and in 8 only with PIRM. In 7 cases out of 38 , where uterus was preserved, ATOM and PIRM were performed for correction of prolapsed uterus and anterior and posterior vaginal wall prolapse. On 29 out of 38 patients correction of prolapsed anterior vaginal wall and uterus was performed with ATOM. In 2 cases from this group of 38 patients only PIRM was performed for posterior vaginal wall prolapse.

Results \& Conclusions: All 62 procedures were performed relatively safely. In 3 cases of ATOM we had perforation of bladder by application of posterior needle and in 2 cases of PIRM we had perforation of rectum. In all 5 cases correction was performed during the operation, mesh was kept in place and postoperative course of treatment had to go without complications.

Short term results, 2 to 3 months after the operation, are very good both for pelvic organ static, and for pelvic function. We also expect long duration of good results.

\section{SS-16}

\section{Laparoscopically assisted vaginal hysterectomy - our experiences}

B. Cvjetičanin, B. Kobal, A. Omahen, M. Barbič, L. Meglič University Clinical Centre, Department of Obstetrics and Gynecology, Ljubljana, Slovenia

Objectives: LAVH is a relatively new operative technique in which laparoscopy as endoscopic technique and classical vaginal surgical route are combined to remove the uterus. It is a micro invasive surgical technique, more friendlily towards patients as laparotomy, which is no more necessary in increasing number of indications

Design\&Methods: The LAVH operative technique was introduced at the Department of Gynecology in Ljubljana in 1994, when the first procedures were done. To evaluate the successfulness of the LAVH technique a specially adapted form is used since 2004 (187 procedures till May 2007).

Results: There were 325 procedures done between 1994 and 2007, and only in the year 2006 as many as 64 . Out of 187 statistically evaluated procedures, 88 were done due to endometrial cancer, 14 due to cervical cancer, and 8 due to combined cancer pathology. LAVH was also performed in 57 patients with myomas or adenomyosis and in 20 with combined benign pathology or pelvic floor disorders. Pelvic lymphadenectomy was performed in 78 patients, vaginoplasty in 21 and cholecystectomy in 3. Laparoscopy was converted into laparotomy in 5 patients.

Conclusion: $\mathrm{LAVH}$ is a safe and towards patient friendly operative technique, suitable to treat a great number of benign and malignant diseases. It is comparable with hysterectomy through laparotomy according to the duration of the procedure, loss of blood and complication rates, but much better regarding the postoperative hospital stay and the use of analgesics. With increasing experiences the number of procedures grows constantly.

\section{SS-17}

Laparoscopic supracervical hysterectomy with the use of LapLoop

M. Ribič-Pucelj, B. Kobal

University Clinical Centre, Department of Obstetrics and Gynecology, Ljubljana, Slovenia

Objective: The purpose of this video is to present the technique of LASH with the use of LapLoop for cutting the uterine corpus from the cervix and the advantages of this device.

Design\&Methods: First LASH was performed at our Department in June 2000 and till June 2007102 LASH were done. Mean patients age was 43,4 years. The most frequent indications were myoma, followed by adenomyosis, while in 3 patients with double uterus the uterus with aplastic cervix was removed due to disturbed menstrual flow and consequent pain and endometriosis. Until 2006 all the procedures were done with ultracision, from 2006 onwards most of the procedures were done with LigaSure. For cutting the uterine corpus from the cervix, the most time consumping and demanding part of the procedure, we used ultracision and exceptionally monopolar needle. In 2006 we started to use for this part of the procedure LapLoop electric wire. After closing the uterine arteries (bipolar coagulation, clips or LigaSure), the electric wire was introduced into the abdominal cavity, put around the cervix above the occluded arteries and hihg monopolar current (at least 120 watts) was applied.

Results: Untill now LapLoop was used in 15 LASH. The average time for cutting was 3 minutes. In comparison with monopolar needle, cutting with LapLoop produced less smoke and in comparison with ultracision knife or hook it was significantly cost effective. The price of reusable wire is 28 EURs.

Conclusion. The advantage of LapLoop is reduced operating time, high precision, great safty and cost effectivnes. 


\section{SS-18}

\section{Laparoscopic uterine artery ligation for treatment of uterine fibroma}

B. Kobal, M. Ribič-Pucelj

University Clinical Centre, Department of Obstetrics and Gynecology, Ljubljana, Slovenia

Background. Retrospective analysis of indications, surgical technique and complications of laparoscopic uterine artery occlusion.

Designand methods. Between January 2005 and July 2006, 11 women with more than 3 myomas exceeding $3 \mathrm{~cm}$ in diameter underwent laparoscopic bilateral uterine artery ligation at the Department of Obstetrics and Gynaecology, University Medical Centre Ljubljana, Slovenia. The mean patient age was 34,6 year (range; 22-48 years) and the mean size of the leading fibroid was $5.6 \mathrm{~cm}$ (range; 3$8 \mathrm{~cm})$. A 4-puncture technique was used for uterine artery isolation at its origin from hypogastric artery. In 7 patients we used the lateral retroperitoneal approach to uterine artery, while in 4 the incision was performed medially at the bifurcation of iliac arteries. Once isolated, titanic endoclips were used to obliterate the vessel. The leading fibroma was then measured sonographically every three months and the fibroid related symptoms were recorded.

Results. The most frequent indications for laparoscopic included meno-metrorrhagia and/or pelvic pain $(n=7$; $63,6 \%)$, reproductive problems $(n=3 ; 27.2 \%)$, and fast growing of fibroids $(n=1 ; 0,9 \%)$. There were no intra or post-operative complications. In one patient a combination of laparoscopic myomectomy and ligation of uterine arteries was performed. The average reduction in size of the leading fibroid after second trimester of follow up was $54 \%$ (range $48-60 \%$ ) in 10 patients, while the improvement of menstrual symptoms and/or pelvic pain was recorded in $8(72,7 \%)$. Second surgical intervention after nine months for continuous growth of fibroids was needed in one patient. Insertion of levonorgestrel uterine device (Mirena- Schering) improved the menstrual symptoms in patients with poor answer to surgical treatment. One patient became spontaneously pregnant and delivered at term.
Conclusions. Laparoscopic uterine artery occlusion is a safe and effective surgical method for selected women with multiple uterine fibroids regarding reduction of fibroid size and improvement of fibroid related symptoms.

\section{SS-19}

\section{Laparoscopic occlusion of ovarian vein} for the treatment of pelvic congestion syndrome: Initial experience and clinical results

\section{B. Kobal}

University Clinical Centre, Department of Obstetrics and Gynecology, Ljubljana, Slovenia

Background. Retrospective analysis of surgical technique and complications and clinical results of laparoscopic ovarian vein occlusion in patients with symptomatic pelvic congestive syndrome.

Patients and methods. Between October 2004 and June 2006, 14 women with chronic pelvic pain and sonographic evidence of pelvic varicosity underwent laparoscopic occlusion of ovarian veins at the Department of Obstetrics and Gynaecology, University Medical Centre Ljubljana, Slovenia. When diagnostic laparoscopy excluded other causes for chronic pelvic pain and the dilated ovarian vessels were revealed, the veins were ligated above the sacroiliac junction, with titanium clips. In four patients $(28,5 \%)$ occlusion was performed bilaterally; while in $10(71.5 \%)$ left ovarian veins were ligated only. Patients were clinically followed for symptoms at two and six months and one year after the procedure.

Results. There were no peri or postoperative complications. Clinical follow-up revealed variable symptomatic relief in two (14.8\%) patients and a total relief of symptoms in eight (57.1\%) patients two months after surgery. Complete remission of pain with or without absence of pelvic varicosities lasted for 12 months of follow up in all eight patients with initial improvement of symptoms.

Conclusion: The laparoscopic transperitoneal approach to bilateral or left sided ligation of ovarian veins outside the small pelvis could be effective in selected patients with pelvic congestion syndrome. 